\title{
Polyclonal Antibody Directed Against Human RANTES Ameliorates Disease in the Lewis Rat Adjuvant-induced Arthritis Model
}

\author{
Debra A. Barnes, ${ }^{\star}$ Jenny Tse, ${ }^{\ddagger}$ Margaret Kaufhold, ${ }^{\ddagger}$ Matt Owen, ${ }^{\ddagger}$ Joe Hesselgesser, ${ }^{*}$ Robert Strieter, ${ }^{\S}$ Richard Horuk, ${ }^{\star}$ \\ and H. Daniel Perez* \\ ${ }^{*}$ Department of Immunology and ${ }^{\ddagger}$ Department of Pharmacology, Berlex Biosciences, Richmond, California 94804; and ${ }^{\S}$ Department of \\ Internal Medicine, University of Michigan Medical School, Ann Arbor, Michigan 48109
}

\begin{abstract}
Adjuvant-induced arthritis (AIA) is one of many animal models of rheumatoid arthritis, a disease characterized by a T-lymphocyte and macrophage cellular infiltrate. We have characterized the development of this disease model with respect to chemokine expression. Increased levels of two chemokines, RANTES, a T-lymphocyte and monocyte chemo-attractant, and $\mathrm{KC}$ a chemoattractant for neutrophils, were found in whole blood and in the joint. Surprisingly, levels of MIP-1 $\alpha$, another T-lymphocyte and monocyte chemoattractant, were unchanged throughout the course of the disease in whole blood and only slightly elevated in the joint. RANTES expression plays an important role in the disease since a polyclonal antibody to RANTES greatly ameliorated symptoms in animals induced for AIA and was found to be as efficacious as treatment with indomethacin, a non-steroidal anti inflammatory. Polyclonal antibodies to either MIP-1 $\alpha$ or KC were ineffective. This is the first report to show the importance of RANTES in the development of AIA. (J. Clin. Invest. 1998. 101:29102919.) Key words: RANTES - MIP-1 $\alpha$ - KC • arthritis • chemokine
\end{abstract}

\section{Introduction}

Rheumatoid arthritis (RA) ${ }^{1}$ is a chronic inflammatory disease characterized by infiltration of the synovial membrane with $\mathrm{T}$ lymphocytes and macrophages and pannus formation over the

D.A. Barnes and J. Tse contributed equally to this work.

Address correspondence to Dr. Debra A. Barnes, Department of Immunology, Berlex Biosciences, 15049 San Pablo Avenue, Richmond, CA 94804-0099. Phone: 510-660-4075; FAX: 510-669-4244; E-mail: debra_barnes@berlex.com J. Tse's current address is Pharmaceutical Division, Bayer Corporation, Berkeley, CA 94701.

Received for publication 3 November 1997 and accepted in revised form 15 April 1998.

1. Abbreviations used in this paper: AIA, adjuvant-induced arthritis; CIA, collagen type II-induced arthritis; DARC, Duffy antigen receptor for chemokines; MIP-1 $\alpha$, macrophage inflammatory protein- $1 \alpha$; RA, rheumatoid arthritis; RANTES, regulated upon activation, normal $\mathrm{T}$ cell expressed and secreted.

J. Clin. Invest.

(C) The American Society for Clinical Investigation, Inc. 0021-9738/98/06/2910/10 \$2.00

Volume 101, Number 12, June 1998, 2910-2919

http://www.jci.org underlying cartilage and bone (1). The pannus is rich in activated macrophages secreting proteases and other inflammatory mediators resulting in destruction of these tissues. In comparison with normal synovial fluid, which is essentially acellular, RA synovial fluid is abundant in neutrophils, macrophages, T lymphocytes, and dendritic cells. It is thought that both humoral immunity and cellular immunity operating at the same time may contribute to the pathology of the disease.

A role for humoral immunity has been proposed based on the presence of rheumatoid factors in the sera of most RA patients (2). Rheumatoid factors are autoantibodies that are directed against the Fc fragment of IgG. Cellular immunity is also thought to be important because of the linkage of RA to certain MHC-encoded T cell restriction elements (e.g., DR4 and DR1 [3, 4]). Examination of RA synovial tissue shows high levels of proinflammatory cytokines IL-1 (5), TNF- $\alpha$ (6-8), IL-6 (6) and growth factors such as GM-CSF (7) and M-CSF (8), but the T cell-derived mediators IL-2 (8), IL-3 (8), IL-4 (9), and TNF- $\beta$ (10) are either absent or present at low levels. These results have led some researchers to conclude that RA is not T cell driven (11) during the chronic phase of the disease.

While the proinflammatory cytokines are thought to play a role in inflammation, there is also a role for those cytokines that are negative immunoregulators and that are inhibitors of inflammation. IL-10 is presumed to repress expression of TNF- $\alpha$ and IL-1 in RA since it was found that a monoclonal antibody that neutralized IL-10 resulted in elevated levels of these proinflammatory cytokines from RA synovial cell cultures (12). More importantly, treatment of mice with anti-IL-10 antibody resulted in a worsening of the clinical score and raised the levels of the chemokines macrophage inflammatory protein-1 $\alpha$ (MIP-1 $\alpha)$ and MIP-2 in the joints of animals induced for type II collagen arthritis (13).

The inflammatory process observed in RA is mediated, in part, by chemotactic factors released by inflamed tissues. The proinflammatory cytokines IL- $1 \beta$ and TNF- $\alpha$ are known to induce expression of small chemotactic proteins (i.e., chemokines) in a number of different cell types. Chemokines are subdivided into two major classes, C-X-C and C-C, depending on the position of the first two cysteines (14). Regulated upon activation, normal T cell expressed and secreted (RANTES; reference 15$)$ and MIP-1 $\alpha(16,17)$ are both members of the C-C chemokine family and have overlapping activities. Both of these chemokines can chemoattract $\mathrm{T}$ lymphocytes, although MIP- $1 \alpha$ preferentially attracts $\mathrm{CD}^{+}$cells $(18,19)$ while RANTES preferentially attracts $\mathrm{CD}^{+} \mathrm{T}$ lymphocytes (20). Both chemokines also attract monocytes $(20,21)$. Recently, MIP- $1 \alpha$, and especially RANTES, have been shown to be able to activate $\mathrm{T}$ lymphocytes and promote $\mathrm{T}$ cell proliferation $(21,22)$. These data suggest that RANTES may be involved in 
the clonal amplification of activated $\mathrm{T}$ cells indicating that RANTES can not only attract activated $T$ cells but also induce the expansion of the activated population at local sites of RANTES production. RANTES and MIP- $1 \alpha$ mediate their effects through specific binding to high affinity receptors expressed on the surface of target cells (23). One of the receptors for these ligands, CCR1, is a member of a growing family of seven transmembrane domain, $G$ protein-linked receptors (24).

Recently, it was found that rheumatoid synovial fibroblasts upregulate RANTES mRNA in response to IL-1 $\beta$, TNF- $\alpha$, and $\gamma$ IFN. Rathanswami et al. (25) demonstrated, by Northern hybridization analysis and ELISA, that cultured synovial fibroblasts isolated from rheumatoid patients were capable of expressing and producing RANTES and other chemokines in response to IL-1 $\beta$. Snowden et al. (26) have used reverse transcriptase-PCR to detect RANTES mRNA in four out of seven synovial tissue samples from rheumatoid arthritis patients. By contrast, osteoarthritis tissue does not express RANTES mRNA (26). These data constitute indirect evidence that RANTES may play a role in RA. The purpose of this study was to determine the role of RANTES in the development of the inflammatory process present in RA. This is only possible with the use of animal models and we have used an adjuvantinduced arthritis (AIA) model in Lewis rats. We also assessed the importance of other chemokines, including MIP- $1 \alpha$ and $\mathrm{KC}$, in the AIA model by comparing control serum-treated animals to those in which chemokine production was blocked by treatment with polyclonal antibody. Our data indicates a specific role for RANTES in the mediation of the inflammatory and destructive aspects of AIA.

\section{Methods}

Antibody. Polyclonal antibody to recombinant human RANTES was prepared by injecting New Zealand white rabbits with purified RANTES ( $250 \mu \mathrm{g}$ per injection) subcutaneously in incomplete Freund's adjuvant followed by subsequent boosts in incomplete Freund's. The serum was collected and titered against immunogen. Titer of the antibody used in this study was $1 / 500,000$ against human RANTES and $1 / 50,000$ for rat RANTES. It did not react with any other chemokine tested including: MIP-1 $\alpha$, MIP-1 $\beta$, MCP-1, KC, MIP-2, IL-1 $\beta$, TNF- $\alpha$, or IL-6. Polyclonal antibody to mouse MIP- $1 \alpha$ was described previously (27) and had a titer of $1 / 1,000,000$ for mouse MIP- $1 \alpha$ and cross reacted with rat MIP- $1 \alpha$ with a titer of $1 / 200,000$. Polyclonal antibody to rat $\mathrm{KC}$ was obtained from PeproTech, Inc. (Rocky Hill, NJ) and had a titer of $1 / 200,000$.

Adjuvant-induced arthritis. All animal studies were approved by the Berlex Biosciences Institutional Review Board. Adult male Lewis rats (160-170 g) were injected with $0.1 \mathrm{ml}$ of CFA containing $10 \mathrm{mg} / \mathrm{ml}$ of Mycobacterium butyricum subcutaneously in the proximal quarter of the tail essentially as described previously (28). The day of injection is designated day 0 . On days 3,5 , and 7 , animals in a given study group were injected with the appropriate antibody or with normal rabbit serum $(0.5 \mathrm{ml} /$ injection per rat $)$ i.p. Animals receiving indomethacin received i.p. injections of $0.5 \mathrm{mg} / \mathrm{kg}$ per day in sterile saline. Vehicle controls for these animals were i.p. injections of sterile saline solution alone. Animals were monitored periodically for body weight, measurement of swelling of each hind paw, degree of redness and flexibility of the rear ankle joints. Each characteristic was then assigned a subjective score of zero to four. The sum of these scores for each animal was determined and the total is designated as the clinical score, as described previously (29).

Radiological score. Whole body radiographs were taken on day
22 after induction and at the end of the study (day 34). Animals were anesthetized with sodium pentobarbital, $20 \mathrm{mg} / \mathrm{kg}$. All radiographs were taken by a Raymax imager (Raymax Medical Corp., Ontario, Canada) at $25 \mathrm{~mA}, 50 \mathrm{kV}$ for anterioposterior or $56 \mathrm{kV}$ for lateral projections with Kodak Memmo Ready Pack film with a $3 \mathrm{~s}$ exposure (Eastman Kodak Co., Rochester, NY). A zero to three subjective grading system was then used to evaluate five different parameters including: degree of swelling, osteoporosis, cartilage loss, erosion, heterotopic ossification, and periosteal new bone formation all as described (29). The radiological score refers to the sum of the subjective scores for each of the above parameters.

Histopathological evaluation. After death, rat paws were resected above the ankle joint and fixed in buffered $10 \%$ formalin. After decalcification in $10 \%$ formic acid, the paws were sectioned longitudinally between digits one and two, and between three and four. These tissue blocks were embedded in paraffin and sectioned longitudinally until the tarsal, metatarsal, and phalangeal joints with adjacent bones and soft tissue were in view. Tissue sections $5-\mu \mathrm{m}$ thick were picked up on glass slides and stained with hematoxylin-eosin and evaluated for morphological changes and cellular infiltrate.

Chemokine determination. Blood was drawn into heparin-containing tubes $(50 \mathrm{U} / \mathrm{ml}$ of blood). An aliquot of $100 \mu \mathrm{l}$ was removed and treated with $100 \mu \mathrm{l}$ of $1 \%$ Triton $\mathrm{X}-100$ in PBS $(1.0 \mathrm{mM}$ $\mathrm{NaH}_{2} \mathrm{PO}_{4}, 8.1 \mathrm{mM} \mathrm{Na} 2 \mathrm{HPO}_{4}, 154 \mathrm{mM} \mathrm{NaCl}, \mathrm{pH}$ 7.2). Chemokines from ankle joints were assayed by first removing the hind feet and distal portion of the leg. The skin was removed and the tissue surrounding the ankle joint was dissected away from the bone. This tissue was placed in a tube containing lysis buffer $(20 \mathrm{mM}$ imidazole$\mathrm{HCl}, \mathrm{pH} 6.8,100 \mathrm{mM} \mathrm{KCl}, 1 \mathrm{mM} \mathrm{MgCl} 2,10 \mathrm{mM}$ EGTA, $1.0 \%$ Triton $\mathrm{X}-100,10 \mathrm{mM}$ NaF, $1 \mathrm{mM}$ sodium molybdate, $1 \mathrm{mM}$ EDTA, $1 \mu \mathrm{g} / \mathrm{ml}$ of leupeptin, aprotinin, and PEFA block) and frozen at $-80^{\circ} \mathrm{C}$. Samples were then thawed and allowed to sit at $4^{\circ} \mathrm{C}$ overnight. An aliquot of the lysate supernatant was removed and spun in a microfuge at $10,000 \mathrm{~g}$ for $5 \mathrm{~min}$ to remove insoluble material. The supernatant was assayed for presence of chemokines. The lysates were also assayed for presence of hemoglobin by measuring the absorbance at $450 \mathrm{~nm}$ compared with whole blood lysate using the method described previously (30).

Chemokines were assayed by the use of ELISA kits (R \& D Systems, Inc., Minneapolis, MN). Because of the high degree of similarity maintained in chemokines across species, kits that used polyclonal antibodies for the detection of either mouse or human chemokines could also be used to detect the rat cognate provided that a standard curve was obtained using known concentrations of rat chemokine. Thus, rat RANTES was assayed using a kit to detect human RANTES except that the standard curve was obtained using rat RANTES (PeproTech, Inc.). MIP- $1 \alpha$ and $\mathrm{KC}$ levels were determined using kits that detect mouse chemokines and, in this case, standard curves were prepared using rat MIP- $1 \alpha$ and rat KC (both from PeproTech, Inc.). Optical densities were read at $450 \mathrm{nM}$ using a $V_{\max }$ kinetic microplate reader (Molecular Devices, Sunnyvale, CA).

\section{Results}

Chemokine production in AIA. Our initial question was aimed at determining whether there was any correlation with the inflammatory and destructive elements of AIA and the appearance of various chemokines in whole blood or joint. A number of different cells make up the cellular infiltrate of the pannus in RA, but the Tlymphocytes and monocytes are postulated to play the most important roles. Therefore, it seemed reasonable to examine the role of chemokines such as RANTES and MIP- $1 \alpha$ that would be expected to attract these cell subsets. In addition we asked whether $\mathrm{KC}$, a chemoattractant for neutrophils that have been postulated to play a role in RA (for review see reference 31) was involved in mediating the disease. 
A

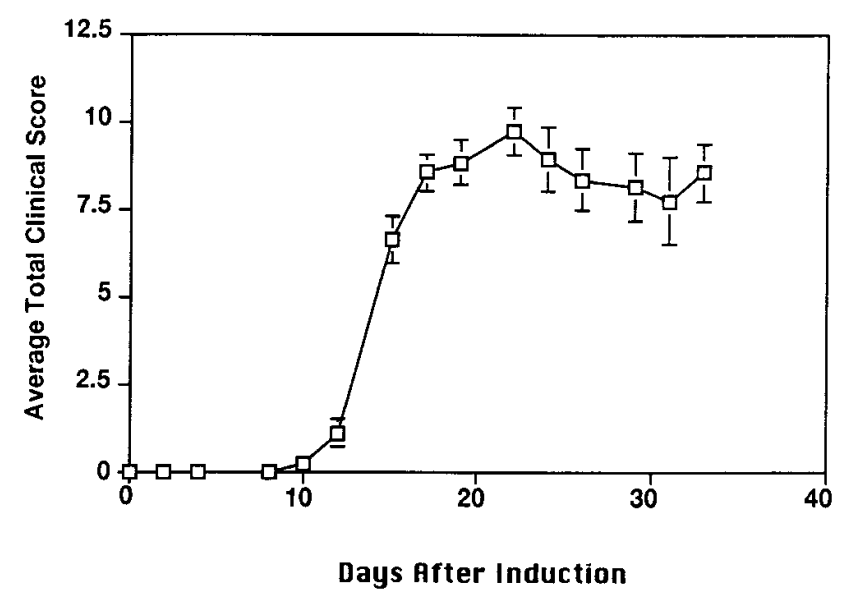

C

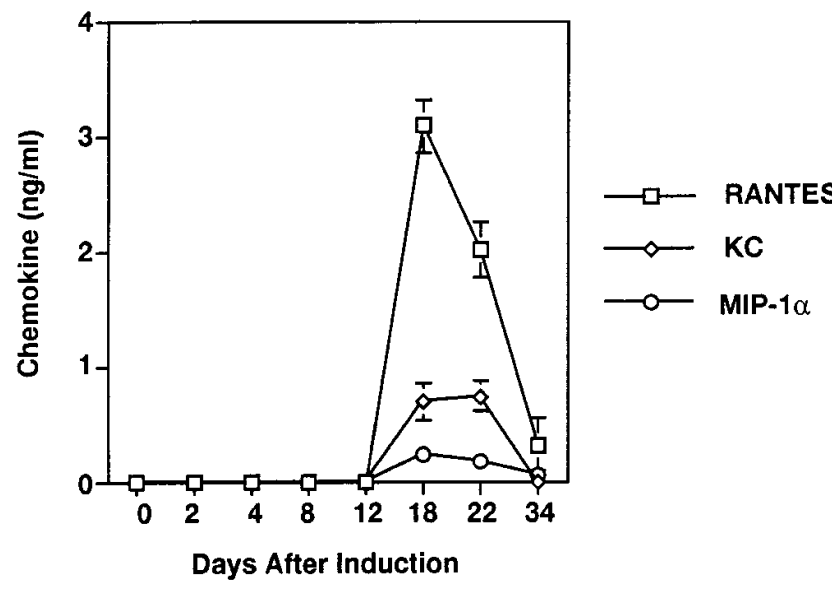

To determine the role of chemokines during the development of AIA, 35 Lewis rats were induced and a simple time course study was performed. In addition, as a control, three age-matched uninduced naive animals were included. Animals were induced on day 0 and five animals each were killed at each time point on days $2,4,8,12,18,22$, and 34 . At the time of death, each animal was assessed for the clinical score. At that time blood samples were collected, $\mathrm{x}$ rays were taken, and joints were prepared. Blood and joint samples were frozen away at $-80^{\circ} \mathrm{C}$ until all samples could be analyzed at the same time at the end of the study. $\mathrm{X}$ rays for all animals were also assessed at the end of the study. As shown in Fig. $1 A$, animals do not show any signs of disease until day 12 . From days 12 to 20, there is a steep increase in clinical score which then plateaus after day 20. The course of AIA is monophasic given that animals, once they become ill, maintain high clinical scores.

The degree of bone destruction with time was also followed using radiographic analysis. Data from the $\mathrm{x}$ rays were analyzed and scored with respect to swelling, osteoporosis, cartilage loss, erosion, heterotopic ossification, and periosteal new

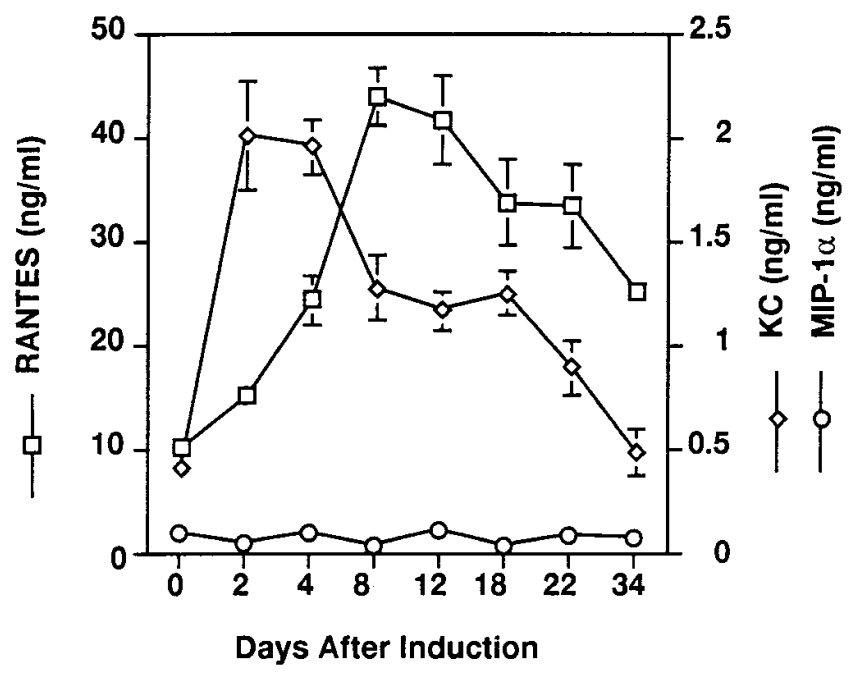

Figure 1. Chemokines and development of AIA. (A) The clinical score during the progression of AIA. Day 0 represents the time of induction. Each time point represents an average of five animals. The SEM is also given for each point. $(B)$ Graph depicting the level of chemokines in whole blood as determined by ELISA. RANTES levels are represented by squares and concentrations are given on the left $y$ axis, MIP- $1 \alpha$ by circles and $\mathrm{KC}$ by diamonds and their concentrations are given on the right $y$ axis. Each point represents the average of five animals and the SEM is given. $(C)$ Graph depicting the level of chemokines in the ankle joint. Graph designations are as in $B$. Each point represents the average of five animals and the SEM is given.

bone formation. Addition of each of these individual numbers gives the radiological score. A study of the radiological score reveals that the bone destruction characteristic of AIA is just detectable at day 22 , but the most deleterious symptoms are not seen until day 34 after induction (data not shown).

As shown in Fig. $1 \mathrm{~B}, \mathrm{KC}$ in whole blood rises immediately after induction and then gradually declines until by the end of the study it has decreased to preinduction levels. MIP- $1 \alpha$, however, never increases and remains at very low levels in the blood (between $50-100 \mathrm{pg} / \mathrm{ml}$ ) throughout the entire study. RANTES is found in normal animals in whole blood at very high levels $(10 \mathrm{ng} / \mathrm{ml})$ probably because of its presence in platelet granules. Whole blood was assayed rather than plasma as it was easier to maintain uniform treatment of samples and bypass the possibility of variable platelet activation. The RANTES levels rise and peak by days 8-12 and then fall. The rise in RANTES levels in the blood just precedes the onset of clinical signs of disease.

Because induction of chemokines in the blood was observed, we were interested to determine whether there would 
also be an increase observed in the ankle joint of the affected animals. As shown in Fig. $1 C$, there is no detectable chemokine in the joint through day 12. Maximal levels were detected at days 18-22, depending on the chemokine. At this point, there is a rise in RANTES as well as KC levels with RANTES being the greater of the two. Both RANTES and KC levels return to undetectable levels by day 34 . There is also a very slight increase in the MIP- $1 \alpha$ levels as compared with those observed in whole blood. The MIP- $1 \alpha$ in the joint is between $200-250$ $\mathrm{pg} / \mathrm{ml}$. It is unlikely that these increases are due to the leakiness of the vasculature surrounding the joint, otherwise one would expect to observe continued high levels of RANTES in the joint on day 34 because blood levels remained high (Fig. $1 \mathrm{~B})$. However, to rule out this possibility, we measured the absorbance of the joint extract at $450 \mathrm{~nm}$ against a standard curve of whole blood. Absorbance at this wavelength gives an indication of the amount of hemoglobin present and allowed us to estimate the degree of contamination in the joint extract. With this approach we calculated that there was $<3 \%$ whole blood contamination of our cell extracts (data not shown). Therefore, at days 18-22 when RANTES is at its peak in the joint, the maximum amount of RANTES present in the joint due to contamination can be no more than $3 \%$ of the value in whole blood (35 ng/ml at days 18-22) and could account for $1 \mathrm{ng} / \mathrm{ml}$. Since the actual measurement is $3 \mathrm{ng} / \mathrm{ml}$, at least $2 \mathrm{ng} / \mathrm{ml}$ must be produced by those cells in the joint. Since MIP- $1 \alpha$ levels are higher in the joint than in the circulation this must be due to production of MIP- $1 \alpha$ in the local environment by resident cells. The fact that by day 34 all chemokine levels return to 0 suggests that RANTES, MIP-1 $\alpha$, and KC may be downregulated in the cells making up the inflammatory response once the destructive process has been initiated.

RANTES is involved in the development of AIA. To determine whether RANTES played any role in the development of AIA, 30 Lewis rats were induced for disease and 5 age-matched naive control animals remained uninduced. Animals were induced on day 0 . On days 3, 5, and 7, 10 animals received normal rabbit serum and ten received a polyclonal antibody directed against recombinant human RANTES. As a positive control, five animals that were induced on day 0 were given indomethacin $(0.5 \mathrm{mg} / \mathrm{kg}$ per day in saline, i.p.) a nonsteroidal anti-inflammatory drug known to ameliorate the clinical effects of AIA through the inhibition of cyclooxygenase (32). These animals received indomethacin on day 0 and throughout the course of the study. Finally, five animals induced on day 0 were given a vehicle control (saline, i.p.) starting on day 0 and continuing through the course of the study. The negative control animals treated with either normal rabbit serum (control for the anti-RANTES-treated group) or with vehicle (control for the indomethacin-treated group) were necessary so that each group of animals would be handled in the same manner. It is well known that differential handling of the animals can lead to ambiguous results. All animals were assessed periodically for clinical score. On days 24 and 34, all animals were $x$ rayed. On day 34 , all of the anti-RANTES, serum control animals and naive animals were killed and joints were prepared for histopathological examination. Indomethacin and vehicle control animals were killed on day 42 .

Clinical scores for this study are shown in Fig. 2. Animals which were treated with control serum or with vehicle had very high cumulative clinical scores. As with the study depicted in Fig. $1 A$, clinical scores begin to rise on day 12 and

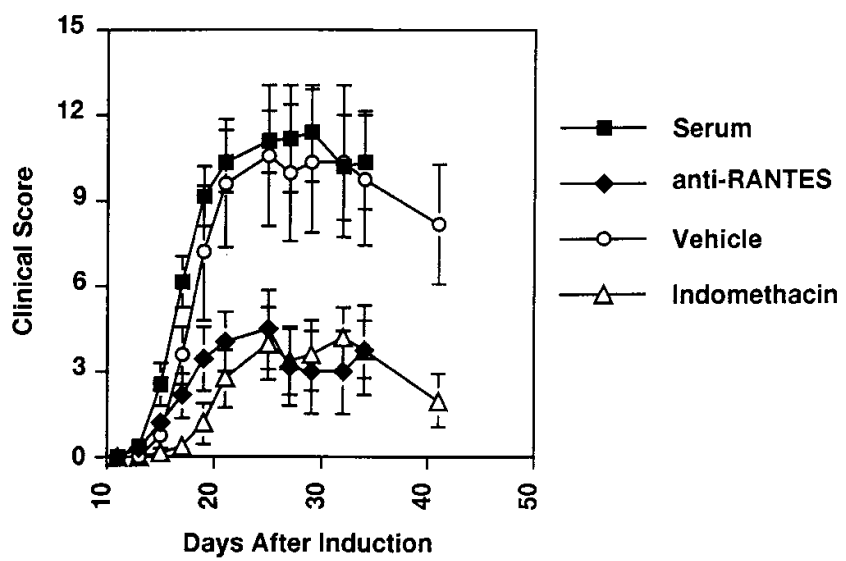

Figure 2. Anti-RANTES and indomethacin treatment ameliorate AIA. There were six animals in each study group. Study groups consisted of anti-RANTES-treated animals (black diamonds), animals given normal rabbit serum (black squares), animals treated with indomethacin (open triangles), and finally animals treated with a vehicle control for indomethacin (open circles). Each point represents the average and the SEM is shown.

peak by day $20-22$. Animals which were treated with either the anti-RANTES antibody or with indomethacin never showed the high clinical scores associated with the disease.

Because the clinical score is associated with the inflammatory response we wanted to determine what effects antiRANTES treatment had on histopathology. Fig. 3 shows the representative histopathological changes in these animals at day 34. First, examination of the synovial joint of naive animals at low magnification reveals that the space between the bones is completely clear (Fig. $3 A$ ). Animals treated with normal rabbit serum have lost the integrity of the joint. This space now shows severe leukocyte infiltration and interstitial edema (Fig. $3 \mathrm{~B}$ ). There is also significant bone erosion and periosteal new bone formation. By comparison the anti-RANTES-treated animals show relatively little infiltration (Fig. $3 C$ ). The synovial lining is discerned at higher magnification (Figs. 3, $D-F$ ). In naive animals (Fig. $3 \mathrm{D}$ ) the synovial cells form a single layer and are flat and quiescent. No leukocyte infiltration is observed. In animals treated with control serum (Fig. $3 E$ ) the synovial lining cells are round to cuboidal, suggesting active proliferation and form a layer two to three cells deep. The underlying connective tissues show infiltration by large numbers of lymphocytes. Irregularly-shaped mesenchymal cells (Fig. $3 E$, arrowhead) are indicative of active differentiation. In the synovial tissues of anti-RANTES-treated rats (Fig. $3 F$ ), fewer leukocytes are present and no active mesenchymal cells are seen. The synovial lining cells, however, are reactive with rounded somata and oval nuclei. Their cytoplasm is often pale and vacuolated. In some areas, these cells appear to be dissociating from the underlying connective tissue.

One of the hallmarks of the AIA model is that it results in severe destruction of the ankle joint with characteristic bone proliferation in soft tissue and concurrent erosion of pre-existing bony structures. This destruction can be assayed by radiographic analysis. An example of representative joints from each of the study groups is shown in Fig. 4, $A-H$. An animal that received the preimmune serum (Fig. 4, $B$ and $F$ ) clearly 

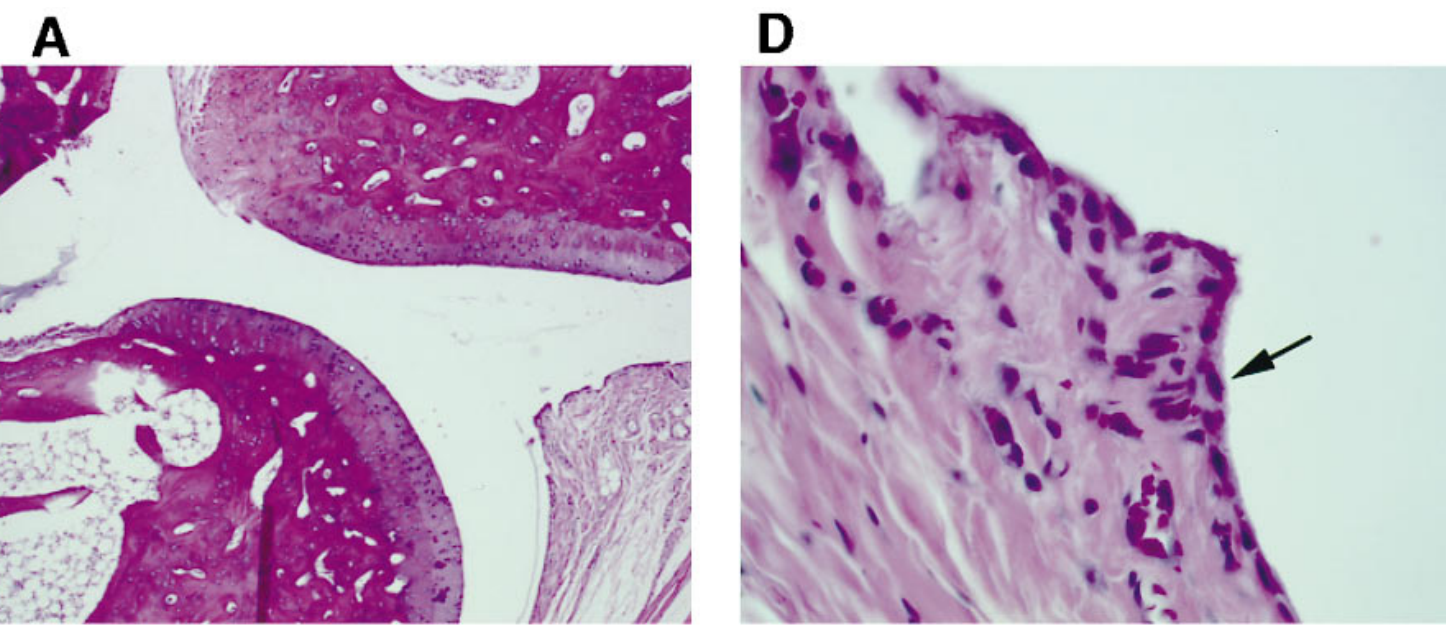

\section{B}

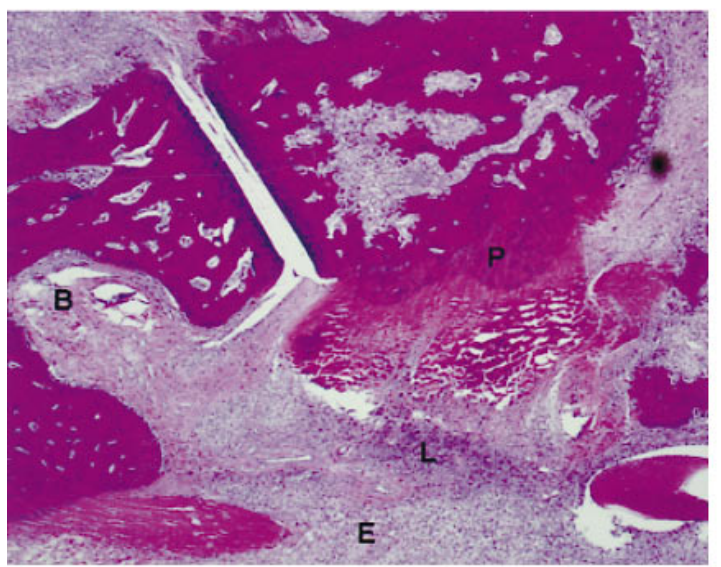

\section{E}
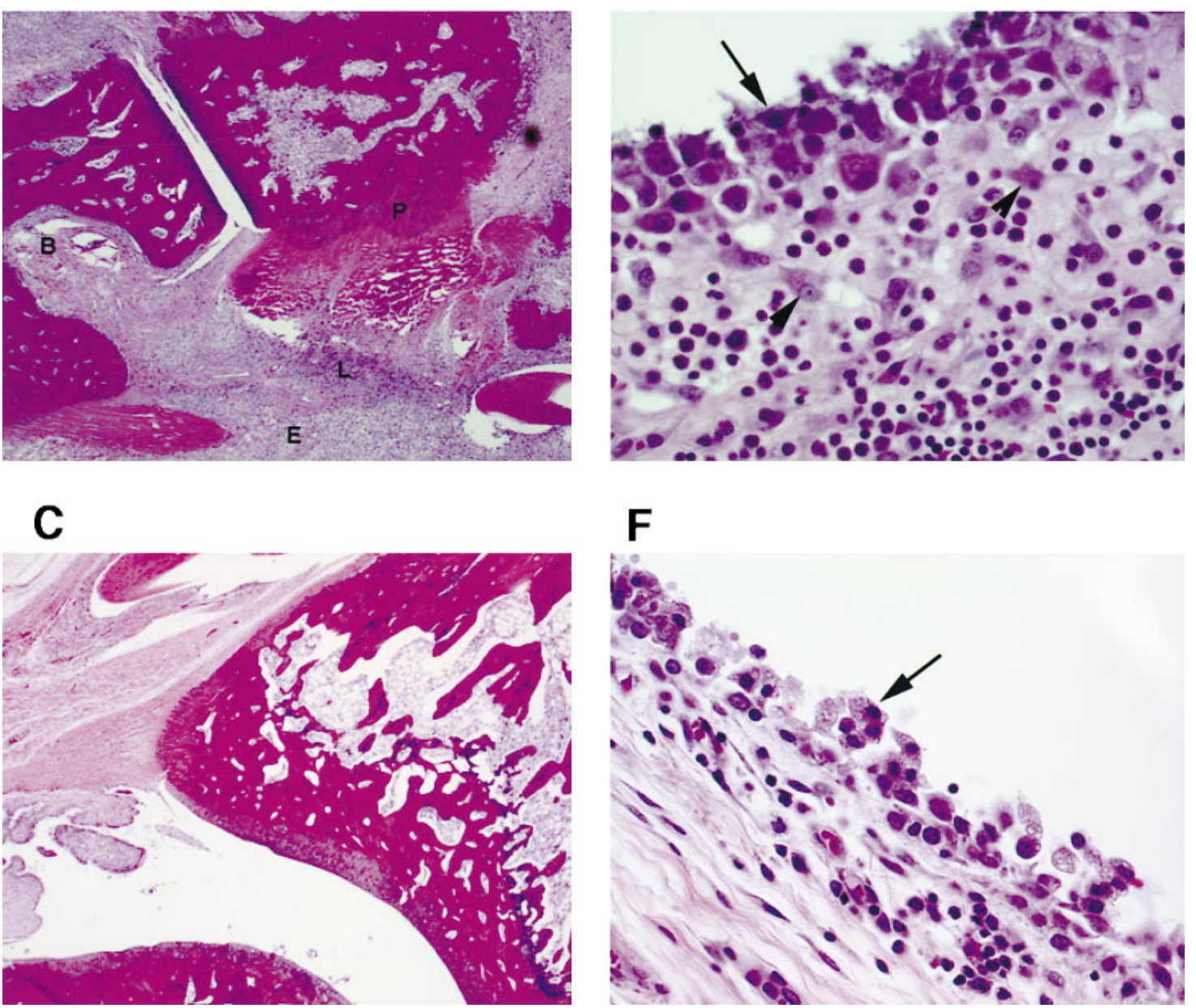

Figure 3. Anti-RANTES treatment reduces the severity of histopathological changes in AIA. $A, B$, and $C$ show a synovial joint at a magnification of 5. $D, E$, and $F$ show synovial tissues at a magnification of $40 . A$ and $D$ show naive rat joint; $B$ and $E$ show an arthritic joint from a rat treated with control serum; $C$ and $F$ show effects of treatment with anti-RANTES antibodies. $B$ shows severe leukocyte infiltration $(L)$, interstitial edema $(E)$, periosteal new bone formation $(P)$, and bone erosion $(B)$. By comparison, the anti-RANTES-treated sample $(C)$ shows very little infiltration. The arrows in $D, E$, and $F$ point to the synovial lining. The arrowheads point to mesenchymal cells.

shows the destruction typically seen using this model. No significant destruction is seen in the naive animals (Fig. 4, $A$ and $E$ ), the anti-RANTES-treated animals (Fig. 4, $C$ and $G$ ), or in the indomethacin control animals (Fig. 4, $D$ and $H$ ).
All of the animals killed in the study were evaluated for bone destruction using $\mathrm{x}$-ray analysis. The summary of the radiological scores are graphed in Fig. 5. At $24 \mathrm{~d}$ there is no significant statistical difference between any of the study groups 


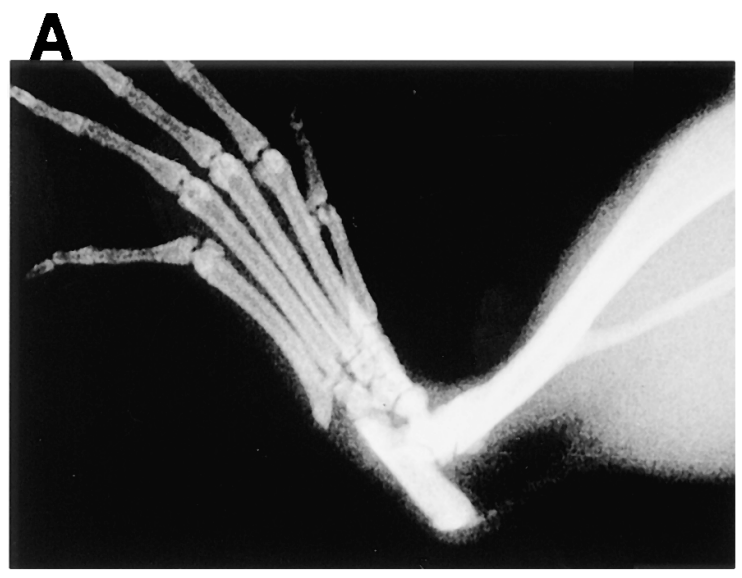

B

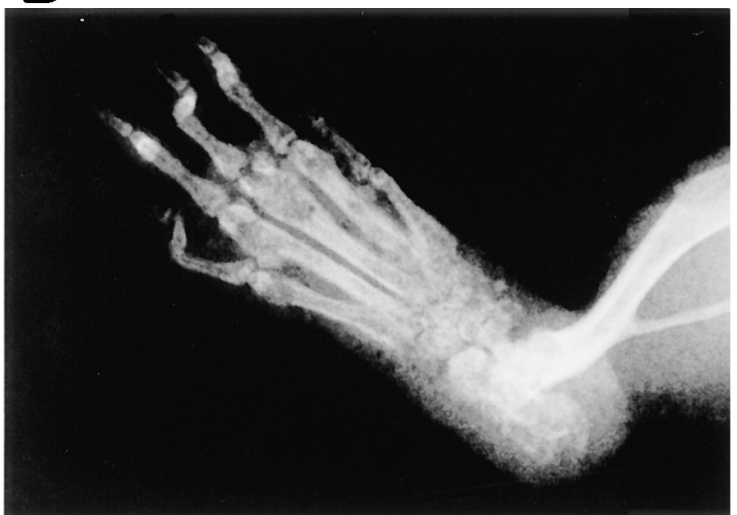

C

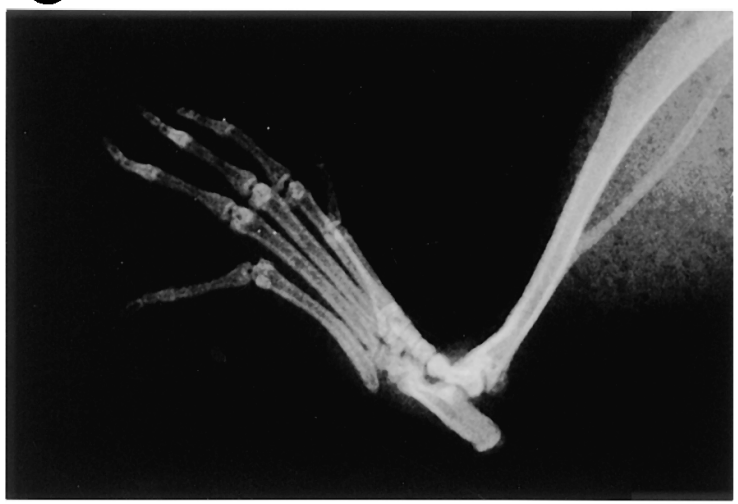

D

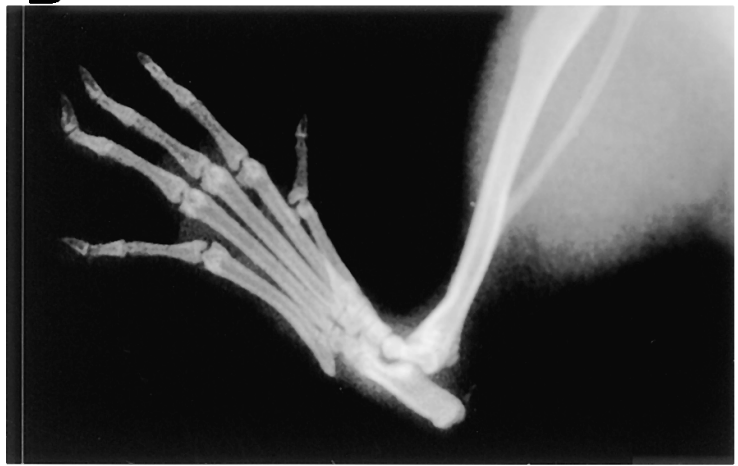

E

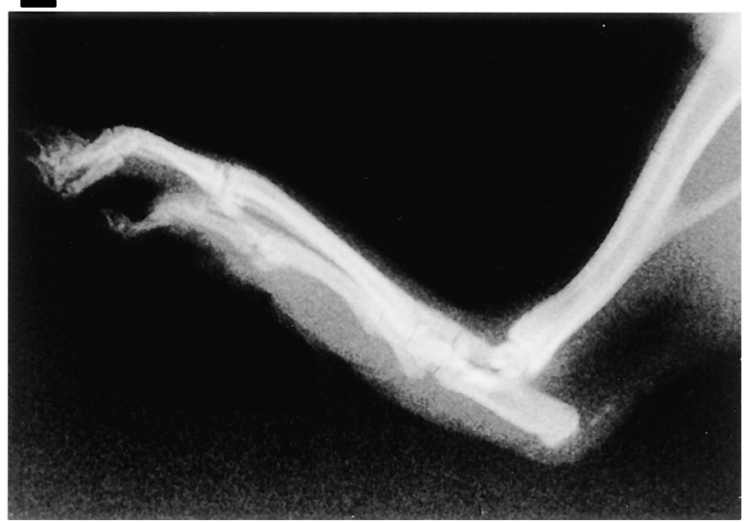

F

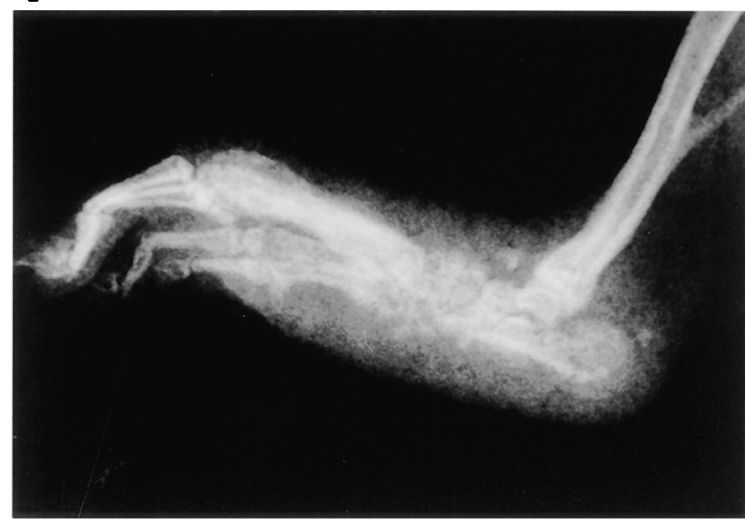

G

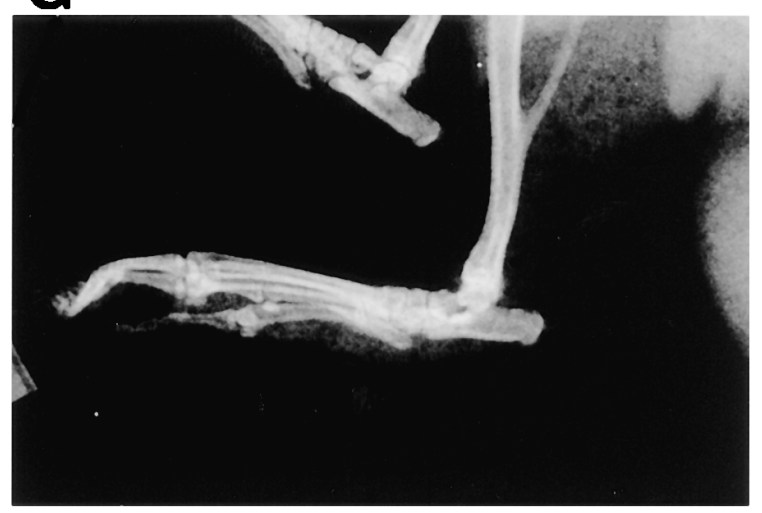

H

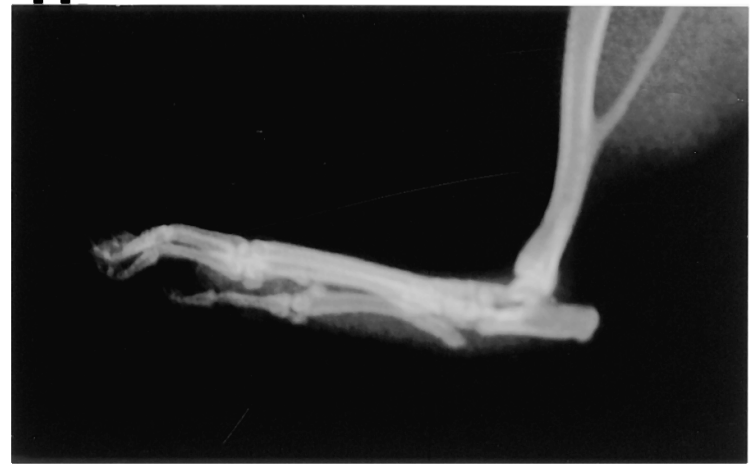

Figure 4. Anti-RANTES treatment reduces the level of bone destruction in AIA. $A, B, C$, and $D$ represent anterioposterior radiographs and $E$, $F, G$, and $H$ represent lateral radiographs. $A$ and $E$ are from naive animal, $B$ and $F$ are from serum control animal, $C$ and $G$ are from antiRANTES treated animal, panel $D$ and $H$ are from an indomethacin-treated animal. Of particular note is the soft tissue swelling and heterotopic ossification. 


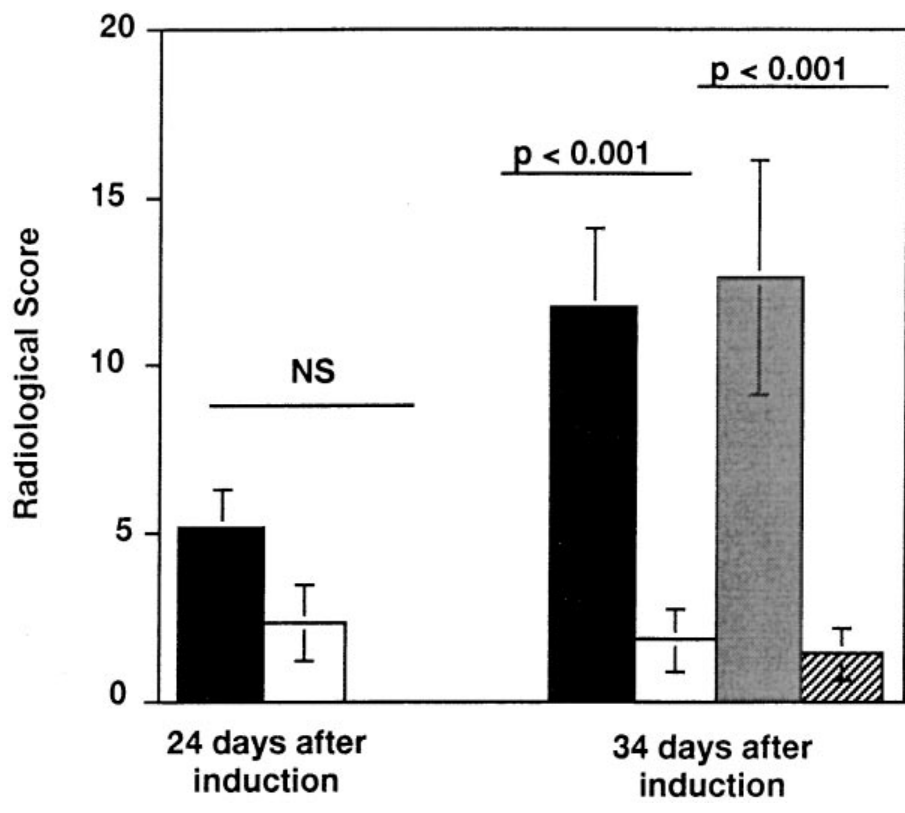

(Fig. 5, only anti-RANTES and serum control group are shown). By day 34, however, destruction has reached very high levels for those animals in the serum control and vehicle control groups. Radiological scores for animals treated with antiRANTES or indomethacin have significantly lower scores. There is no statistical difference between the anti-RANTEStreated group and those given indomethacin.

These data strongly suggest that RANTES is important in the development of AIA and that pretreatment of the animals before onset of disease with an antibody directed against RANTES prevents the inflammation and destruction associated as efficiently as a known nonsteroidal anti-inflammatory, indomethacin.

Specific chemokine involvement in AIA. Because we had shown a role for RANTES in the development of AIA, and because RANTES and MIP- $1 \alpha$ are ligands for the chemokine receptor CCR1 (24), we wanted to determine whether MIP-1 $\alpha$ was involved in the pathophysiology of the disease. To answer this question various animal study groups were treated with polyclonal antibodies directed against either RANTES or MIP-1 $\alpha$. Two groups of 16 animals were injected either with anti-RANTES, or with anti-MIP-1 $\alpha$ all as described in the previous study. In addition, 16 animals were injected with normal rabbit serum. 3 animals out of the 16 in both the antiMIP-1 $\alpha$ and serum control group did not develop disease and were not included in the study. To maintain consistency, three animals from the anti-RANTES group that did not develop disease were also excluded from the study. Clinical scores were determined periodically. Half of the animals from each group were killed on day 22 and the remaining half were killed on day 34. At those times they were subjected to radiography.

Clinical scores from this study are given in Fig. $6 \mathrm{~A}$. Again, anti-RANTES is found to ameliorate the symptoms of AIA. Polyclonal antibody to MIP- $1 \alpha$ had no effect on amelioration of disease. This is interesting given the overlapping activities of these two chemokines. Polyclonal antibody to $\mathrm{KC}$ was also tested in this manner and had no effect on the course of the disease (data not shown).
The fact that anti-RANTES treatment before appearance of clinical symptoms has an effect on disease progress is shown most clearly by examining the level of this chemokine in the ankle joint itself. As shown in Fig. 6 B, RANTES levels in the serum control joint are high as expected at day 22 and drop back down again at day 34 , the end of the study. The antiRANTES-treated animals, however, have much reduced levels of RANTES in the joints. These data correlate with the lower clinical scores (Fig. $6 \mathrm{~B}$ ) and with a reduced radiological score (data not shown) observed in the anti-RANTES animals and is a result of the prevention of the cellular infiltrate from reaching the target joint in the first place.

\section{Discussion}

The availability of animal models of human diseases makes possible the identification and analysis of factors involved in pathogenesis. The usefulness of a model is determined by its relative similarity to human disease, its reproducibility and its predictability with respect to responsiveness to therapeutic agents in comparison to human disease. Two widely used animal models of rheumatoid arthritis are collagen type II-induced arthritis (CIA) and AIA.

We have used the AIA model of RA in the Lewis rat to examine the role RANTES plays in the development of this disease. Unfortunately, there is no animal model available that perfectly mimics the course and features of human rheumatoid arthritis. Both AIA and CIA, however, show peripheral joint involvement, erosion, pannus formation, and $\mathrm{T}$ cell dependence (for review see reference 33 ) similar to that observed in human disease.

In this study, we determined the levels of the chemokines RANTES, MIP- $1 \alpha$, and KC in AIA animals. We found very little change in the MIP- $1 \alpha$ levels in either whole blood or in the joint. The levels of $\mathrm{KC}$ in whole blood were elevated immediately after induction through day 4 and then were found to slowly decrease until they resumed preinduction levels by day 34. These data measure the total amount of $\mathrm{KC}$ in blood. 

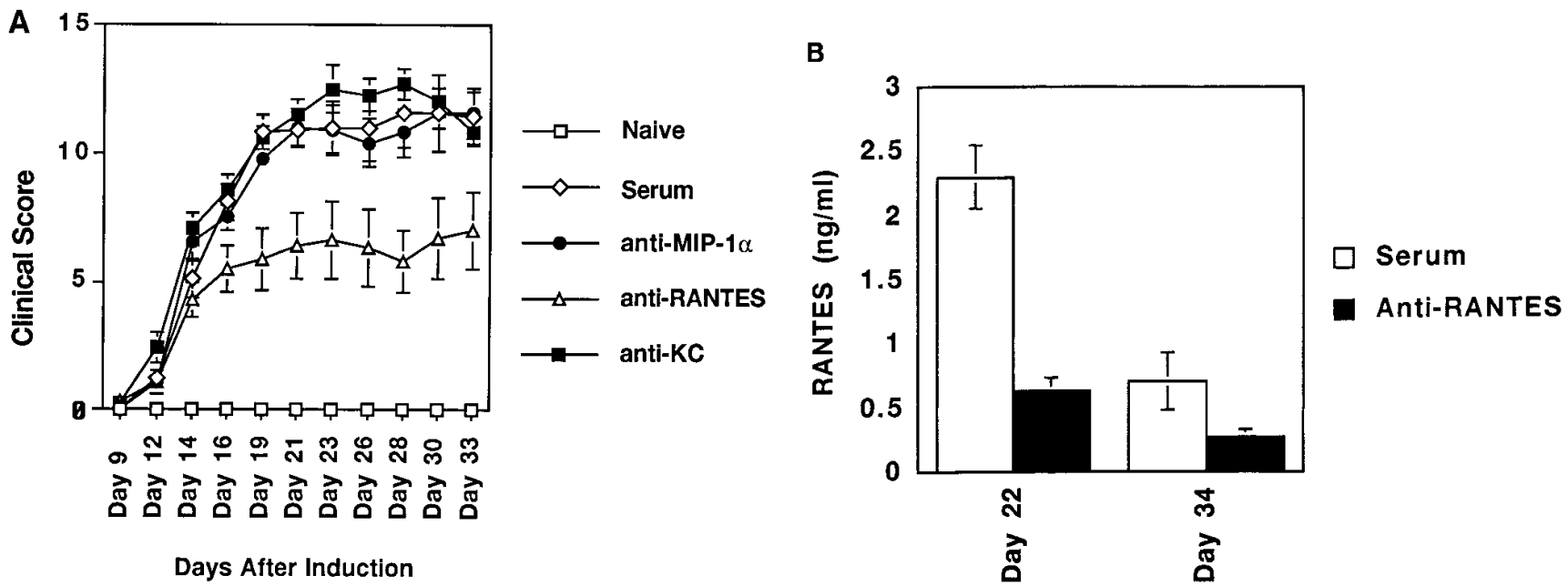

Figure 6. Development of AIA is chemokine specific. $(A)$ Lewis rats were induced for AIA and injected on days 3, 5 , and 7 with polyclonal antibody to RANTES (open triangles), MIP-1 $\alpha$ (black circles), KC (black squares), or normal rabbit serum (open diamonds). Each point represents the mean average. The SEM is also given. $(B)$ RANTES levels in the joint of anti-RANTES-treated animals and serum-treated animals.

The circulating levels of $\mathrm{KC}$ in plasma are undetectable (Barnes, D.A., unpublished observation). This discrepancy may be due to the binding of $\mathrm{KC}$ to the Duffy antigen receptor for chemokines (DARC) which is found in abundance on the surface of red blood cells and binds this chemokine (34). It has been suggested that DARC serves as a receptor sink for a number of chemokines, including RANTES, and may be involved in clearing of these potent peptides (35). If this receptor plays a role in the modulation of RA, by clearing chemokines from the blood, one might expect that individuals that are Duffy negative would have more devastating disease. In fact, African Americans who are in large part Duffy negative have a much lower incidence of RA than the Caucasian population (36). This is primarily a result of the fact that African Americans do not generally carry the DR1 or DR4 markers. DR1 and DR4 are variants of the HLA-D locus and are highly correlated with appearance of rheumatoid factors in patients with RA. It would be interesting to determine whether Duffy negative individuals that are diagnosed with RA have more severe disease than those that are Duffy positive.

RANTES has an entirely different profile than $\mathrm{KC}$ and levels of this chemokine are not elevated until days 8-12, the time just before the onset of inflammation as measured by the clinical score. While the cellular source of this circulating RANTES is still unclear, the levels in circulation must be higher than can be removed by DARC, otherwise one would predict that an antibody that binds RANTES would have no effect since RANTES would be bound to DARC and unavailable to the antibody. Furthermore, because antibody was given in only three injections (days 3, 5, and 7) and would be predicted to be cleared from the animal after $3 \mathrm{~d}$, it suggests that the RANTES generated in the circulation during the day 8-12 time period is critical to disease development.

Previously, it was shown that when mice were induced for CIA and passively immunized with antibodies directed against MIP-1 $\alpha$ or MIP-2 they had significantly decreased severity of disease (13). Unlike Kasama et al. (13), we did not find that treatment with antibody to MIP- $1 \alpha$ had any ameliorating effect on disease. However, we used a different animal model in this study. The CIA model has a much longer time course $(>60 \mathrm{~d})$ than the AIA model and the rise in clinical score is much more gradual. In addition, anti-MIP- $1 \alpha$ treatments in the form of $\mathrm{F}(\mathrm{ab})_{2}$ fragments were provided throughout the study whereas in the AIA model, anti-RANTES treatment was limited to three injections before onset. Nevertheless, the actual amount of MIP- $1 \alpha$ detected at peak levels in the joints of afflicted CIA animals was quite low $(200 \mathrm{pg} / \mathrm{ml})$ and there appeared to be a very low level constitutive expression of MIP-1 $\alpha(100 \mathrm{pg} / \mathrm{ml})$. These data agree well with our findings in the AIA model in the rat. The induction of MIP-2 observed in the CIA model was much greater, from $<100 \mathrm{pg} / \mathrm{ml}$ in naive animals to $800 \mathrm{pg} / \mathrm{ml}$ in CIA induced animals.

Our finding that antibodies to RANTES but not to MIP- $1 \alpha$ ameliorate disease in this RA model are interesting, particularly since both of these CC chemokines bind to similar receptors, CCR1 and CCR5 (23). How, then, can we explain our data? Interestingly, Cook et al. (37) have examined the in vivo biologic role of MIP- $1 \alpha$ in mice in which the gene encoding the chemokine has been disrupted. They showed that mice homozygous for MIP- $1 \alpha$ mutant $(-/-)$ were resistant to Coxsackie virus-induced myocarditis but wild-type $(+/+)$ mice were not. Obviously in this case MIP- $1 \alpha$ and RANTES have entirely separate effects. Further evidence that these two chemokines have distinct biological effects comes from findings by Karpus et al. (27) who have shown that antibodies to MIP-1 $\alpha$ ameliorate an EAE model of multiple sclerosis in the mouse. We have repeated these studies and not only confirm their data but show that antibodies to RANTES have no effect in this model (Barnes, D., and J. Tse, data not shown). Thus, it is possible that our data can be explained by assuming that RANTES effects in the AIA model of RA are produced by action through an as yet uncloned chemokine receptor or through a distinct signaling pathway, that MIP- $1 \alpha$ cannot trigger, through an existing receptor. In line with these specula- 
tions, it is interesting that at least one group of investigators (21) has reported that T cells stimulated with RANTES and with MIP-1 $\alpha$ produce an increase in intracellular $\mathrm{Ca}$ at low $\mathrm{nM}$ concentrations. However, at higher concentrations RANTES but not MIP-1 $\alpha$ produces a second $\mathrm{Ca}^{2+}$ transient that is more sustained than the first and is not desensitized by pretreatment with MIP-1 $\alpha$. These data show that RANTES can activate a novel chemokine receptor that is not sensitive to MIP- $1 \alpha$ and that activation of this receptor may play a role in this model of RA.

There is clear evidence that RANTES and MIP- $1 \alpha$ have distinctly different effects on T cells and monocytes, the major cell types involved in RA. For example a number of reports have demonstrated that RANTES affects the directed migration of $\mathrm{CD}^{+} \mathrm{T}$ cells and upregulates integrin expression, all effects that would culminate in the extravasation of $\mathrm{T}$ cells to sites of inflammation $(18,21)$. In contrast, it appears that MIP$1 \alpha$ is mainly effective in inducing the directed migration of $\mathrm{CD}^{+}$, but not $\mathrm{CD}^{+}$, $\mathrm{T}$ cells. In addition to these cell-specific effects, RANTES is also about 10 times more potent than MIP-1 $\alpha$ in inducing $\mathrm{T}$ cell migration (18). Taken together, these data provide a partial explanation for the effects of RANTES on the RA model that we have investigated here and may speak for a specific effect of this chemokine in the human disease.

It has recently been shown that a human monocyte chemoattractant protein-1 (MCP-1) antagonist greatly ameliorated adjuvant arthritis-induced disease in a mouse MRL-lpr genetic background (38). MCP-1 is a chemokine with the ability to attract monocytes (39), the precursors of macrophages. The MRL-lpr disease model has an earlier onset than that observed in the rat AIA model, although, as with the AIA model, onset is rapid and the development from no detectable symptoms to peak clinical score occurs within a matter of days (generally from day 5-10). In these experiments, an MCP-1 peptide (amino acids 9-76) was synthesized and found to compete with MCP-1 for binding to monocytes. This antagonist, when given continually throughout the course of disease, resulted in decreased inflammation. However, the MCP-1 antagonist was given only through day 15 (after inflammation had already peaked) and then withdrawn. After withdrawal the disease progressed until swelling reached the same level as that observed in the untreated animals.

This is a very different pattern from what we observed with the RANTES antibody treatment. In our study anti-RANTES treatment ended on day 7. By day 11 one would predict that all of the rabbit polyclonal antibody had disappeared. Since peak inflammation is observed by day 18, the data suggest that early expression of RANTES in circulation is one of the critical steps involved in development of AIA. RANTES may be involved in initiating the inflammatory response because this chemokine has the potential to play key roles as both a chemoattractant and as an immunomodulator, since it also serves to activate and expand clonal $\mathrm{T}$ cell populations. It should also be noted that studies by Plater-Zyberk et al. (40) using an altered form of RANTES, met-RANTES, which acts as a CCR1 antagonist were also able to show efficacy in an animal model of RA. In their studies, delivery of the antagonist i.p. three times per week through day 21 resulted in the delay of onset and amelioration of CIA in DBA/1 mice. These data constitute additional proof for the role of RANTES in the development of disease.
If RANTES directs the initial response, what roles do other chemokines play in the development of AIA? It may be that the chemokines MIP- $1 \alpha$ and MCP-1 are expressed later in the course of disease and are involved in the destructive process of arthritis by their actions on the appropriate cell types once the initial inflammatory signal has been given.

We show here that RANTES is expressed in whole blood and in the joint of AIA animals and that antibody to RANTES can prevent development of disease. Thus, these data strongly support the concept that RANTES plays a pivotal role in the pathogenesis of RA. These potent proinflammatory effects and chemoattraction for $\mathrm{T}$ lymphocytes and monocytes makes RANTES an attractive candidate for therapeutic intervention. Furthermore, since RANTES binds to and mediates its biologic effects through the CCR1 receptor, this makes this receptor an important target for intervention therapy in RA and provides evidence in support of the notion that CCR1 antagonists will be useful therapeutics in the treatment of RA. Other useful therapeutic approaches could, for example, take the form of inhibitors of the cellular expression of RANTES. Finally, an anti-RANTES treatment regimen has the potential to serve not only as an anti-inflammatory agent, but also as a disease modifying antirheumatic drug.

\section{Acknowledgments}

The authors would like to thank Ms. Baby Martin-McNulty, Mr. Cornell Mallari and Ms. Wendy Fraser for technical assistance and Dr. Jean Merrill for a critical reading of this manuscript.

\section{References}

1. Harrison's Principles of Internal Medicine, 13th Ed. 1994. K.J. Isselbacher, E. Braunwald, J.D. Wilson, J.B. Martin, A.S. Fauci, and D.L. Kasper, editors. McGraw-Hill Inc., New York. 1437-1443.

2. Waller, M. 1969. Methods of measurement of rheumatoid factor. Ann. NY Acad. Sci. 168:5-16.

3. Stastny, P. 1978. HLA-D and Ia antigens in rheumatoid arthritis and systemic lupus erythematosus. Arthritis Rheum. 21:S139-S143.

4. Woodrow, T.C., F.E. Nichol, and G. Zaphiropoulos. 1981. DR antigens and rheumatoid arthritis: a study of two populations. Br. Med. J. 283:1287-1288.

5. Fontana, A., H. Hentgartner, K. Fehr, P.J. Grob, and G. Cohen. 1982. Interleukin-1 activity in the synovial fluid of patients with rheumatoid arthritis. Rheumatol. Int . 2:49-56.

6. Houssiau, F.A., J.-P. Devogelaer, J. van Damme, C. Nagant de Deuxchaisnes, and J. van Snick. 1988. Interleukin-6 in synovial fluid and serum of patients with rheumatoid arthritis and other inflammatory arthritides. Arthritis Rheum. 31:784-788.

7. Xu, W.D., G.S. Firestein, R. Taetle, K. Kaushansky, and N.J. Avaifler. 1989. Cytokines in chronic inflammatory arthritis. II. Granulocyte-macrophage colony-stimulating factor in rheumatoid synovial effusions. J. Clin. Invest. 83: 876-882.

8. Firestein, G.S., W.-D. Xu, K. Townsend, D. Broide, J. Alvaro-Gracia, A. Glasebrook, and N.J. Zvaifler. 1988. Cytokines in chronic inflammatory arthritis. I. Failure to detect T cell lymphokines (interleukin-2 and interleukin-3) and presence of macrophage colony-stimulating factor in rheumatoid synovitis. $J$. Exp. Med. 168:1573-1586.

9. Miossec, P., M. Naviliat, A.D. D'Angeac, J. Sany, and J. Bancherau. 1990. Low levels of interleukin-4 and high levels of transforming growth factor b in rheumatoid synovitis. Arthritis Rheum. 33:1180-1187.

10. Saxne, T., M.A. Palladino, D. Heinegard, N. Talal, and F.A. Wollheim. 1988. Detection of tumor necrosis factor as but not tumor necrosis factor b in rheumatoid arthritis synovial fluid and serum. Arthritis Rheum. 3:1041-1045.

11. Firestein, G.S., and N.J. Zvaifler. 1990. How important are T cells in chronic rheumatoid synovitis? Arthritis Rheum. 33:768-773.

12. Katsikis, P., C. Chu, F.M. Brennan, R.N. Maini, and M. Feldmann. 1994. Immunoregulatory role of interleukin 10 (IL-10) in rheumatoid arthritis. $J$. Exp. Med. 179:1517-1527.

13. Kasama, T., R.M. Strieter, N.W. Lukacs, P.M. Lincoln, M.D. Burdick, and S.L. Kunkel. 1995. Interleukin-10 expression and chemokine regulation during the evolution of murine type II collagen-induced arthritis. J. Clin. Invest. 95:2868-2876. 
14. Oppenheim, J.J., C.O.C. Zachariae, N. Mukaida, and K. Matsushima. 1991. Properties of the novel proinflammatory supergene "intercrine" cytokine family. Annu. Rev. Immunol. 9:617-648.

15. Schall, T.J., J. Jongstra, B.J. Bradley, J. Dyer, J. Jorgensen, C. Clayberger, M. Davis, and A.M. Krensky. 1988. A human T cell-specific molecule is a member of a new gene family. J. Immunol. 141:1018-1025.

16. Brown, K.D., S.M. Zurawski, T.R. Mosmann, and G. Zurawski. 1989. A family of small inducible proteins secreted by leukocytes are members of a new superfamily that includes leukocytes and fibroblast-derived inflammatory agents, growth factors and indicators of various activation processes. J. Immunol. 142:679-687.

17. Zipfel, P.F., J. Balke, S.G. Irving, K. Kelly, and U. Siebenlist. 1989. Mitogenic activation of human T-cells induces two closely related genes which share structural similarities with a new family of secreted factors. J. Immunol. 142:1582-1590.

18. Taub, D.T., K. Conlon, A.R. Lloyd, J.J. Oppenheim, and D.J. Kelvin. 1993. Preferential migration of activated $\mathrm{CD} 4^{+}$and $\mathrm{CD} 8^{+} \mathrm{T}$ cells in response to MIP-1 $\alpha$ and MIP-1ß. Science. 260:355-358.

19. Schall, T.J., K. Bacon, D.R. Camp, J.W. Kaspari, and D.V. Goeddel. 1993. Human macrophage inflammatory protein a (MIP-1 $\alpha$ ) and MIP-1 $\beta$ chemokines attract distinct populations of lymphocytes. J. Exp. Med. 177:18211826 .

20. Schall, T.J., K.B. Bacon, K.J. Toy, and D.V. Goeddel. 1990. Selective attraction of monocytes and T lymphocytes of the memory phenotype by cytokine RANTES. Nature. 347:669-671.

21. Bacon, K.B., B.A. Premack, P. Gardner, and T.J. Schall. 1995. Activation of dual T cell signaling pathways by the chemokine RANTES. Science. 269: 1727-1730.

22. Taub, D.D., S.M. Turcovski-Corrales, M.L. Key, D.L. Longo, and W.J. Murphy. 1996. Chemokines and T lymphocyte activation. I. $\beta$ chemokines costimulate human T lymphocyte activation in vitro. J. Immunol. 156:2095-2103.

23. Horuk, R. 1994. Molecular properties of the chemokine receptor family. Trends Pharmacol. Sci. 15:159-165.

24. Neote, K., D. DiGregorio, J.Y. Mak, R. Horuk, and T.J. Schall. 1993. Molecular cloning, functional expression, and signaling characteristics of a C-C chemokine receptor. Cell. 72:415-425.

25. Rathanaswami, P., M. Hachicha, M. Sadick, T.J. Schall, and S.R. McColl. 1993. Expression of the cytokine RANTES in human rheumatoid synovial fibroblasts. J. Biol. Chem. 268:5834-5839.

26. Snowden, N., A. Hajeer, W. Thomson, and B. Ollier. 1994. RANTES role in rheumatoid arthritis. Lancet. 343:547-548.

27. Karpus, W.J., N.W. Lukacs, B.L. McRae, R.M. Strieter, S.L. Kunkel, and S.D. Miller. 1995. An important role for the chemokine macrophage in- flammatory protein-1 $\alpha$ in the pathogenesis of the T cell-mediated autoimmune disease, experimental autoimmune encephalomyelitis. J. Immunol. 155:50035010 .

28. Pearson, C.M. 1956. Development of arthritis, periarthritis and periostitis in rats given adjuvants. Proc. Soc. Exp. Biol. Med. 91:95-101.

29. Ackerman, N.R., W.H. Rooks, L. Shott, H. Genant, P. Maloney, and E. West. 1979. Effects of Naproxen on connective tissue changes in the adjuvant arthritic rat. Arthritis Rheum. 22:1365-1374.

30. Horuk, R., A.W. Martin, W.Z.L. Schweitzer, A. Gerassimides, H. Guo, Z. Lu, J. Hesselgesser, H.D. Perez, J. Kim, J. Parker, et al. 1997. Expression of chemokine receptors by subsets of neurons in the central nervous system. $J$. Immunol. 158:2882-2890.

31. Edwards, S.W., and M.B. Hallett. 1997. Seeing the wood for the trees: the forgotten role of neutrophils in rheumatoid arthritis. Immunol. Today. 18 $320-324$.

32. Meade, E.A., W.L. Smith, and D.L. DeWitt. 1993. Differential inhibition of prostaglandin endoperoxide synthase (cyclooxygenase) isozymes by as pirin and other nonsteroidal antiinflammatory drugs. J. Biol. Chem. 268:66106614

33. Oliver, S.J., and E. Brahn. 1996. Combination therapy in rheumatoid arthritis: the animal model perspective. J. Rheumatol. 23(Suppl. 44):56-60.

34. Horuk, R., A. Martin, J. Hesselgesser, T. Hadley, Z.-H. Lu, Z.-X. Wang, S.C. Peiper. 1996. The Duffy antigen receptor for chemokines: structural analysis and expression in the brain. J. Leukocyte Biol. 59:29-38.

35. Darbonne, W.C., G.C. Rice, M.A. Mohler, T. Apple, C.A. Hebert, A.J. Valente, and J.B. Baker. 1991. Red blood cells are a sink for Interleukin-8, a leukocyte chemotaxin. J. Clin. Invest. 88:1362-1369.

36. Marsh, W.L. 1975. Present status of the Duffy blood group system. CRC Crit. Rev. Clin. Lab. Sci. 5:387-412.

37. Cook, D.N., M.A. Beck, T.M. Coffman, S.L. Kirby, J.F. Sheridan, I.B. Pragnell, O. Smithies. 1995. Requirement of MIP-1 $\alpha$ for an inflammatory response to viral infection. Science. 269:1583-1585.

38. Gong, J.-H., L.G. Ratkay, J.D. Waterfield, and I. Clark-Lewis. 1997. An antagonist of monocyte chemoattractant Protein 1 (MCP-1) inhibits arthritis in the MRL-lpr mouse model. J. Exp. Med. 186:131-137.

39. Yoshimura, T., E.A. Robinson, S. Tanaka, E. Appella, J.-I. Kuratsu, and E.J. Leonard. 1989. Purification and amino acid analysis of two human gliomaderived monocyte chemoattractants. J. Exp. Med 169:1449-1459.

40. Plater-Zyberk, C., A.J. Hoogewerf, A.E.I. Proudfoot, C.A. Power, and T.N.C. Wells. 1997. Effect of a CC chemokine receptor antagonist on collagen induced arthritis in DBA/1 mice. Immunol. Lett. 57:117-120.

41. Finney, D.J. 1964. Statistical methods in biological assay. Hafner Publishing Company, Inc., New York, NY. 25-30. 